\title{
Acute Coronary Syndrome Occurring in a Sub-Saharan Patient Treated with a Reduced Dose of Sorafenib for an Unresectable Hepatocellular Carcinoma: Case Report
}

\author{
Antonin Wilson Ndjitoyap Ndam ${ }^{1,2 *}$, Murielle Lema Helles ${ }^{1}$, Pierre Mathurin Kowo ${ }^{1}$, \\ Mazou Temgoua Ngou', Etienne Atenguena², Blaise Mounpou1, Aicha Yap', \\ Tangie Ngek Larry', Paul Talla ${ }^{2}$, Jerôme Boombhi', Alain Menanga1, \\ Firmin Ankouane Andoulo', Elie Claude Ndjitoyap Ndam ${ }^{1}$ \\ ${ }^{1}$ Department of Internal Medicine and Specialities, Faculty of Medicine and Biomedical Sciences, University of Yaounde I, \\ Yaounde, Cameroon \\ ${ }^{2}$ Hepatogastroenterology and Oncology Unit, Yaoundé General Hospital, Yaounde, Cameroon \\ Email:*tonindam3@yahoo.fr, murielle.lema@gmail.com, kowomathurinp@yahoo.fr, neurotemgoua@yahoo.fr, \\ atenguenatien2002@gmail.com, blaisemounpou@yahoo.fr, aicha_mya@yahoo.fr, ngeklatan@yahoo.com,p_talla@yahoo.fr, \\ boombhijerome@yahoo.fr, apmcbl@hotmail.com, ankouaneandoulo@yahoo.com, ecndam@yahoo.fr
}

How to cite this paper: Ndam, A.W.N., Helles, M.L., Kowo, P.M., Ngou, M.T., Atenguena, E., Mounpou, B., Yap, A., Larry, T.N., Talla, P., Boombhi, J., Menanga, A., Andoulo, F.A. and Ndam, E.C.N. (2020) Acute Coronary Syndrome Occurring in a Sub-Saharan Patient Treated with a Reduced Dose of Sorafenib for an Unresectable Hepatocellular Carcinoma: Case Report. Open Journal of Gastroenterology, 10, 45-51. https://doi.org/10.4236/ojgas.2020.103005

Received: February 2, 2020

Accepted: March 9, 2020

Published: March 12, 2020

Copyright $\odot 2020$ by author(s) and Scientific Research Publishing Inc. This work is licensed under the Creative Commons Attribution International License (CC BY 4.0).

http://creativecommons.org/licenses/by/4.0/

\section{(c) (i) Open Access}

\begin{abstract}
Sorafenib is a chemotherapy used as first line treatment in primary liver cancers. It is an oral antiangiogenic treatment which reduces the progression of the tumor. Some mild or severe side effects have been reported among which some uncommon cardiac events: myocardial infarction and cardiogenic stroke. Sorafenib treatment remains expensive and not frequently used in Sub-Saharan countries. Thus, few studies have described its side effects in this milieu. We report a case of acute coronary syndrome occurring in a 75-year-old female patient, without cardiovascular risks factors, after nine months of sorafenib chemotherapy at a reduced dose for an unresectable hepatocellular carcinoma in a Sub-Saharan Africa country. The management was conducted by cardiologists, in collaboration with gastroenterologists and oncologists. We decided to completely stop sorafenib chemotherapy. We observed a reduction of the pain 48 hours after her admission, and a regression of electrocardiographic signs after 8 days. In conclusion, the sorafenib treatment can be associated with cardiac events despite the dose reduction.
\end{abstract}

\section{Keywords}

Acute Coronorary Syndrome, Sorafenib, Hepatocellular Carcinoma, Hepatitis C Virus, Yaoundé 


\section{Introduction}

Sorafenib is a chemotherapy drug used in case of some liver, thyroid and kidney cancers. It is an oral Vascular Endothelial Growth Factor Receptor (VEGFR) tyrosine kinase inhibitor. It has been validated, since 2007, as the first line of treatment for unresectable hepatocellular carcinoma [1] [2] [3]. It is an antiangiogenic therapy which inhibits tumor angiogenesis, and induces tumor cell apoptosis [4]. The recommended posology is $400 \mathrm{mg}$ twice daily. It is generally well tolerated, but can be responsible of adverse effects [3]. Most of them are mild: fatigue, loss of appetite, hand-foot syndrome, nausea and vomiting, diarrhea. But some cases of severe side effects are reported. Cardiovascular events are possible, but not frequent. They include arterial hypertension, congestive heart failure, cardiomyopathy, acute myocardial infarction, and cardiogenic stroke [5] [6] [7] [8] [9]. These side effects are underestimated, but can lead to the death of the patient before the cancer disease. In Sub-Saharan Africa, sorafenib remains expensive for the population. For this reason, few studies have described side effects which can occur with this treatment. We herein report the case of an acute coronary syndrome in a 75-year-old female patient with low cardiovascular risks, treated with a reduced dose of sorafenib for an unresectable hepatocellular carcinoma complicating a hepatitis $\mathrm{C}$ induced compensated liver cirrhosis.

\section{Case Presentation}

A 75-year-old Cameroonian female patient, presented at the Emergency Department with a sudden onset constrictive precordial chest neither pain, without irradiation, with neither fever nor cough. The first electrocardiogram showed a ST segment elevation in V1 to V4 with Q waves (Figure 1). At the same time, biological exams showed: Troponine I $=2.47 \mathrm{ng} / \mathrm{ml}$ (normal value $<0.02$ ) and CPK-MB $=80.3 \mathrm{IU} / \mathrm{l}$ (normal value $<25$ ) were significantly elevated. We made the diagnosis of an acute coronary syndrome of the antero-septal and apical segments.

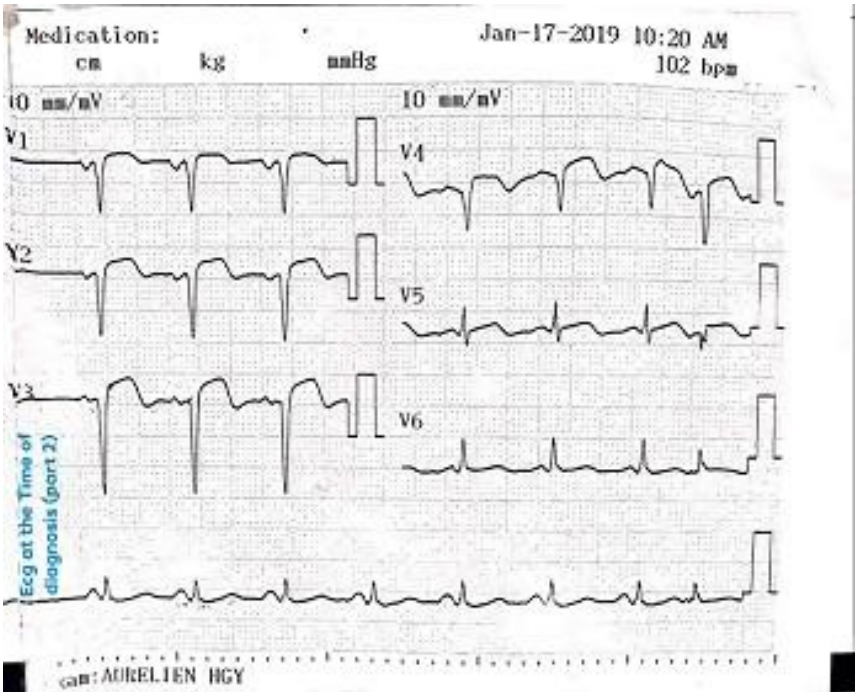

Figure 1. The ECG at the time of the diagnosis. 
Nine months prior to admission, she was diagnosed with a hepatocellular carcinoma. This tumor was discovered in a context of right upper quadrant abdominal pains. Abdominal ultrasound showed a large mass of the liver, measuring $110 \mathrm{~mm}$, and localized on segments VII and VIII. Biphasic CT-scan showed that this heterogenous mass was hyper vascularised at the arterial phase, with a wash-out at the portal phase. There was neither portal thrombosis nor ascites. The Alphafoeto-protein level was elevated at $1241 \mathrm{ng} / \mathrm{ml}$ (normal value was less than 15 $\mathrm{ng} / \mathrm{ml}$ ). There was no history of alcohol consumption. At the time of diagnosis, the patient was good looking, and classified stage one of World Health Organization (WHO) performance status. We neither found ascites nor encephalopathy. The prothrombin time (69\%) and serum Albumin (32 g/l) were mildly low. Alanine aminotransferase (102 IU/l) and aspartate aminotransferase (140 IU/l) were elevated. The hepatitis $C$ virus antibodies and viral load were positive. Gastroscopy did not find any endoscopic signs of portal hypertension. We retained as diagnosis a hepatocellular carcinoma stage B of the BCLC (Barcelona Cancer Liver Center) classification, complicating a hepatitis $\mathrm{C}$ induced compensated liver cirrhosis Child-Pugh A [10].

In accordance with BCLC classification, this tumor was non-resectable [9]. The gastroenterologist proposed palliative treatment by chemo-embolization of the mass. But this therapy was not available in our setting. We thus, administered a treatment based on an oral-chemotherapy with sorafenib $400 \mathrm{mg}$ twice daily, associated with tramadol $50 \mathrm{mg}$ every 8 Hours in case of right upper quadrant abdominal pains. After one month of treatment, the patient presented with a hand-foot syndrome reaction. It was managed by local treatment without significant regression. After three months of treatment, we decided to reduce the posology to only $200 \mathrm{mg}$ twice daily. We observed an improvement of hand-foot syndrome. She also complained of fatigue, hair loss and thinning skin with this treatment.

After nine months of oral chemotherapy by sorafenib, she complained of sudden onset constrictive chest pain. The patient had no past history of cardiac stroke, and low cardiovascular risks. Biological exams showed only a mild dyslipidemia (HDL Cholesterol $=33 \mathrm{mg} / \mathrm{l}$, normal value $40-75 \mathrm{mg} / \mathrm{l}$ ). The cardiac ultrasonography showed an akinesia of the anteroseptal and apical segments.

The management was done by cardiologists in collaboration with gastroenterologists and oncologists. We proposed enoxaparine 8000 IU every 12 hours, clopidogrel $75 \mathrm{mg}$ every 24 hours, aspirine $100 \mathrm{mg} 24$ hourly, rosuvastatine $10 \mathrm{mg}$ 24 hourly, ramipril $2.5 \mathrm{mg}$ every 24 hours, tramadol $100 \mathrm{mg}$ every 8 hours, omeprazole $40 \mathrm{mg}$ every 24 hours. We decided to completely stop sorafenib and we observed a reduction of the pain 48 hours after her admission, and a regression of biological and electrocardiographic signs after 8 days (Figures 2-4).

She stayed without sorafenib treatment and any other chemotherapy regimen. Lenvatinib and second-line treatments like Regorafenib, Ramucirumab and Cabozantinib were not yet available in our country [11] [12] [13]. She died 19 months after the diagnosis of hepatocellular carcinoma, and ten months after the acute coronary syndrome in a context of liver insufficiency. 


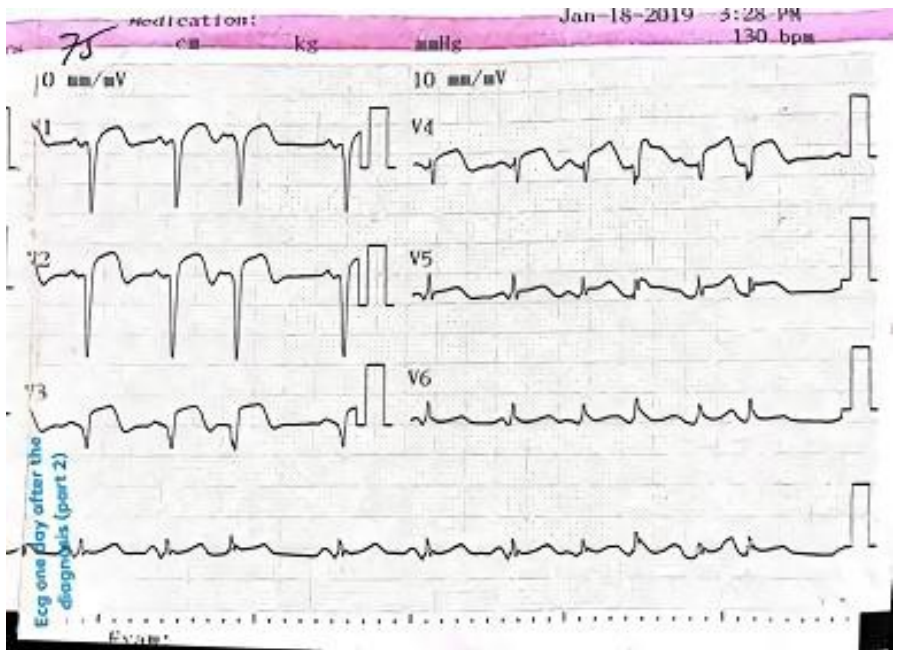

Figure 2. The ECG 24 hours after the diagnosis.

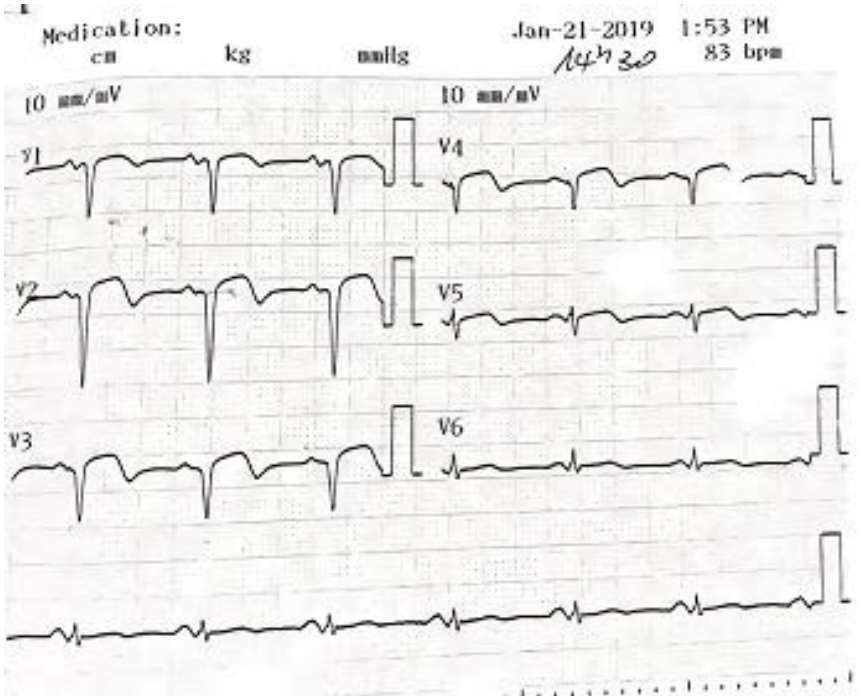

Figure 3. The ECG 5 days after the diagnosis.

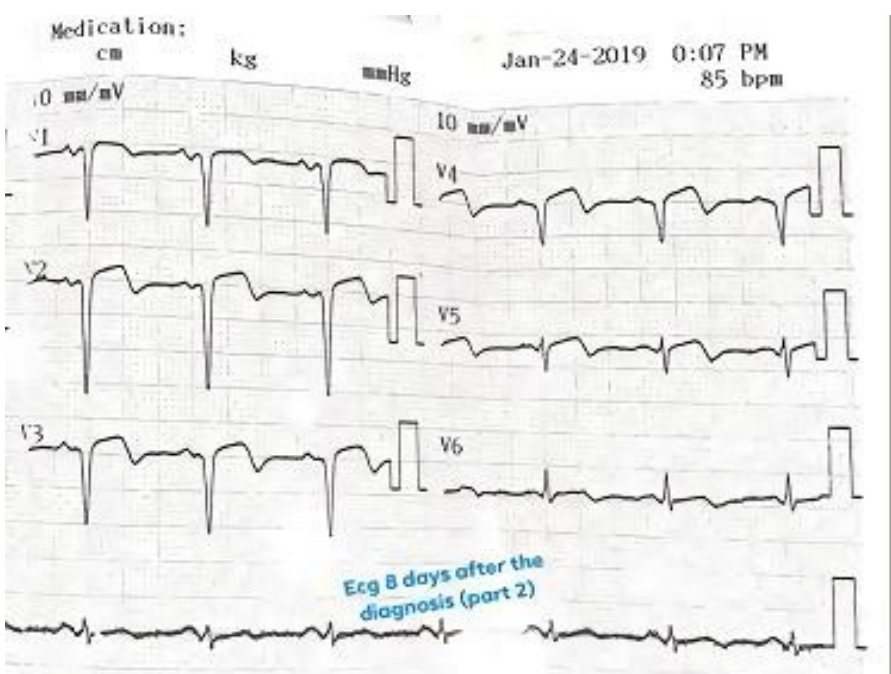

Figure 4. The ECG 8 days after the diagnosis. 


\section{Discussion}

The onset of this acute coronary syndrome, in this patient with low cardiovascular risks and no past history of ischemic heart disease, is thought to be associated with sorafenib which is a VEGFR tyrosine kinase inhibitor. Thus, it reduces cellular proliferation by destroying vascularization. Some cases of myocardial infarction have been reported in literature. Two mechanisms have been described: coronary spasm and coronary artery sclerosis [5] [6] [9]. In our patient, it is probably an artery sclerosis. Coronary artery spasm does not lead to ST-segment elevation. This chemotherapy induced hypercontraction of smooth coronary muscle. The cessation of sorafenib and administration of Calcium-channel blockers and nitrates improve symptoms. But relapse occurred after resumption of sorafenib [6].

The patient complained of a severe hand-foot syndrome before myocardial infarction. We reduced the posology, but the treatment remained efficient [2]. It could be interesting to evaluate if the hand-foot reaction can be used to predict the risk of cardiac stroke. We noted that acute coronary syndrome occured despite the reduction of the dose of sorafenib to $200 \mathrm{mg}$ twice a day due to the severity of hand-foot syndrome. This suggests that's the myocardial infarction can occur despite the reduction of doses.

In literature, the cardiac toxicity of sorafenib has been described, but few publications reported cases of acute coronary syndrome. Many reasons could explain this observation. The first one is the fact that it is an uncommon adverse effect. Another one is the life expectancy with the primary liver tumor. Perhaps patients die too early from their cancer. The objective of the sorafenib in hepatocellular carcinoma is to increase the median survival from 8 to 11 months. Our patient was at 9 months of treatment and thus within the median survival period for patients on this therapy.

A similar case of a sudden cardiac death in a patient with a reduced-dose of sorafenib treatment for a hepatocellular carcinoma has also been reported in Italy in 2017. The man was aged of 51-year-old without cardiovascular risks factors. He received a full dose of sorafenib for an unresectable hepatocellular carcinoma. Three weeks after the introduction of this treatment, he complained of diarrhea and hand-foot syndrome. They reduced the dose to $400 \mathrm{mg}$ per day until the unset of the sudden cardiac attack. This attack happened after 8 months of treatment and led to the death of the patient within 10 minutes [7].

We can't miss out the fact that chronic liver disease which led to cirrhosis and hepatocellular carcinoma was induced by infection with the hepatitis $C$ virus. A chronic infection by the hepatitis $C$ virus can lead to some systemic manifestations which include heart affections without any cancer [14] [15] [16]. It is a potential risk factor for myocardial infarction in our patient independently of sorafenib.

\section{Conclusion}

We report a case of an acute coronary syndrome occurring in a 75-year-old sub-Saharan female patient treated with a reduced dose of sorafenib for an un- 
resectable hepatocellular carcinoma. This cardiovascular event is probably associated with chemotherapy, and we observed an improvement with stopping of sorafenib treatment. More studies need to be done to better explain this association between sorafenib, myocardial infarction, and the pathogenic mechanisms.

\section{Consent for Publication and Ethical Consent}

The patient gives her authorization for this presentation. She remains anonymous. Our institution does not need an ethical approval.

\section{Conflicts of Interest}

The authors declare no conflicts of interest regarding the publication of this paper.

\section{References}

[1] Llovet, J.M., Ricci, S., Mazzaferro, V., Hilgard, P., Gane, E., Blanc, J.F., de Oliveira, A.C., Santoro, A., Raoul, J.L., Forner, A., Schwartz, M., et al. (2008) Sorafenib in Advanced Hepatocellular Carcinoma. The New England Journal of Medicine, 359, 378-390. https://doi.org/10.1056/NEJMoa0708857

[2] Wang, S.X., Byrnes, A., Verma, S., Pancoast, J.R. and Rixe, O. (2010) Complete Remission of Unresectable Hepatocellular Carcinoma Treated with Reduced Dose of Sorafenib. A Case Report. Target Oncology, 5, 59-63. https://doi.org/10.1007/s11523-010-0133-x

[3] Ozenne, V., Paradis, V., Pernot, S., Cateinau, C., Vullierme, M.P., Bouattour, M., Valla, D., Farges, O. and Degos, F. (2010) Tolerance and Outcome of Patients with Unresectable Hepatocellular Carcinoma Treated with Sorafenib. European Journal Gastroenterology Hepatology, 22, 1106-1110. https://doi.org/10.1097/MEG.0b013e3283386053

[4] Liu, L., Cao, Y., Chen, C., Zhang, X., McNabola, A., et al. (2006) Sorafenib Blocks the RAF/MEK/ERK Pathway, Inhibits Tumor Angiogenesis, and Induces Tumor Cell Apoptosis in Hepatocellular Carcinoma Model PLC/PRF/5. Cancer Research, 66, 11851-11858. https://doi.org/10.1158/0008-5472.CAN-06-1377

[5] Pantaleo, M.A., et al. (2012) Development of Coronary Artery Stenosis in a Patient with Metastatic Renal Cell Carcinoma Treated with Sorafenib. BMC Cancer, 12, Article No. 231. https://doi.org/10.1186/1471-2407-12-231

[6] Arima, Y. and Ogawa, H. (2009) Sorafenib-Induced Acute Myocardial Infarction Due to Coronary Artery Spasm. Journal of Cardiology, 54, 512-515. https://doi.org/10.1016/j.jjcc.2009.03.009

[7] Calistri, L., Cordopatri, C., et al. (2017) Sudden Cardiac Death in a Patient with Advanced Hepatocellular Carcinoma with Good Response to Sorafenib Treatment: A Case Report with Literature Analysis. Molecular and Clinical Oncology, 6, 389-396. https://doi.org/10.3892/mco.2017.1132

[8] Wu, C. and Shemisa, K. (2017) Sorafenib-Associated Heart Failure Complicated by Cardiogenic Shock after Treatment of Advanced Stage Hepatocellular Carcinoma. Case Report in Cardiology, 2017, Article ID: 7065759. https://doi.org/10.1155/2017/7065759

[9] Naib, T., Steingart, R.M. and Chen, C.L. (2011) Sorafenib-Associated Multivessel Coronary Artery Vasospasm. Herz, 36, 348-351.

https://doi.org/10.1007/s00059-011-3444-5 
[10] Pons, F., Varela, M. and Josep, M. (2005) Staging Systems in Hepatocellular Carcinoma. HPB, 7, 35-41. https://doi.org/10.1080/13651820410024058

[11] Bakouny, Z., Assi, T., El Rassy, E. and Nasr, F. (2019) Second-Line Treatments of Advanced Hepatocellular Carcinoma: Systematic Review and Network Meta-Analysis of Randomized Controlled Trials. Journal of Clinical Gastroenterology, 53, 251-261. https://doi.org/10.1097/MCG.0000000000001160

[12] Bruix, J., Qin, S., Merle, P., Granito, A., Huang, Y.H., Bodoky, G., et al. (2017) Regorafenib for Patients with Hepatocellular Carcinoma Who Progressed on Sorafenib Treatment (RESORCE): A Randomized, Double-Blind, Placebo-Controlled, Phase 3 Trial. The Lancet, 389, 56-66. https://doi.org/10.1016/S0140-6736(16)32453-9

[13] Kudo, M. (2017) Lenvatinib in Advanced Hepatocellular Carcinoma. Liver Cancer, 6, 253-263. https://doi.org/10.1159/000479573

[14] Petta, S. (2017) Hepatitis C and Cardiovascular: A Review. Journal of Advanced Research, 8, 161-168. https://doi.org/10.1016/j.jare.2016.06.001

[15] Shoeib, O., Ashmawy, M., et al. (2018) Association between Coronary Artery Disease and Hepatitis C Virus Seroposititivity. EMHJ, 24, 618-623. https://doi.org/10.26719/2018.24.7.618

[16] Salam, R.A., Baher, N., et al. (2016) Prevalence of Hepatitis C Virus Seropositivity and Its Impact on Coronary Artery Disease among Egyptian Patients Referred for Coronary Angiography. Cardiology Research and Practice, 2016, Article ID: 1623197. https://doi.org/10.1155/2016/1623197 